\title{
28 Research Square \\ Quality Of Life And Its Influencing Factors In Adult Patients Receiving Maintenance Hemodialysis In China
}

\author{
Mengna Guo \\ Hangzhou Normol University \\ Zhandong Jia \\ Fanli Meng ( $\sim$ mfl1206@126.com ) \\ Hangzhou Normol University \\ Qing Guo \\ Zhejiang Chinese Medical University \\ Yanyan Hong \\ Hangzhou Normol University \\ Yan Ma \\ Hangzhou Normol University
}

Ningbo Municipal Hospital of TCM, Affiliated Hospital of Zhejiang Chinese Medical University

\section{Research Article}

Keywords: Maintenance hemodialysis, Quality of Life, Self-feelings of health status, Influencing factors, Health promotion

Posted Date: February 17th, 2022

DOI: https://doi.org/10.21203/rs.3.rs-1337435/v1

License: (c) (7) This work is licensed under a Creative Commons Attribution 4.0 International License. Read Full License 


\section{Abstract}

Introduction: The purpose of this study was to investigate the health status and quality of life of patients receiving maintenance hemodialysis(MHD) in coastal areas of Zhejiang Province and explore the influencing factors of quality of life.

Methods: This study selected MHD patients from four hospitals in the coastal area of Zhejiang province as the research objects and collected the sociodemographic characteristics, objective physiological condition, self-feelings of health status and quality of life through questionnaire surveys. One-way analysis of variance and multiple linear regression analysis were used to analyze the factors affecting the quality of life of MHD patients.

Results: A total of 140 MHD patients were included in this study. The results showed that the average score of general physical health was $42.89 \pm 9.86$, general psychological health was $46.71 \pm 7.42$, kidney disease-related symptoms was $77.95 \pm 10.82$, kidney disease impact was $64.98 \pm 12.03$ and kidney disease-related burden was 32.01 \pm 19.35 . There were significant differences in subscale scores and total scores according to different gender, age, education level, employment status, marital status, self-feelings of physical health, self-feelings of appetite status and self-feelings of sleep quality status groups $(P<0.05)$. The results of multiple linear regression analysis showed that patients with poor self-feelings of physical health status, poor self-feelings of appetite status and poor self-feelings of sleep quality had worse quality of life $(P<0.05)$.

Conclusion: Self-feelings of physical health status, self-feelings of appetite status and self-feelings of sleep quality are the main factors affecting the quality of life of MHD patients. Medical staff need to pay more attention to the self-feelings of health status of MHD patients, so as to improve the quality of life of MHD patients.

\section{Introduction}

Maintenance hemodialysis(MHD) is the main alternative treatment method for patients with end-stage renal disease(ESRD). With the extension of treatment time, a large amount of harmful substances accumulate in the patient's body, which will cause a series of complications and adverse reactions, causing the patient to suffer from fatigue, itching, muscle cramps, joint pain and other symptoms for a long time [1-2]. In recent years, the number of MHD patients has been increasing year by year and the incidence is showing a younger trend in China [3]. Studies have shown that the total prevalence of MHD was about 414/1,000,000 as of 2018 and the number of patients receiving hemodialysis has exceeded 500,000 in China [4]. The physical health, mental health, sleep condition, daily activity ability and social function of MHD patients are obviously poorer than those of healthy people [5]. Notably, reduced quality of life was strongly associated with higher risk of death and hospitalization in MHD patients [6]. With the transformation of modern medical models, the quality of life is getting more and more attention. This means that medical treatment not only needs to extend the survival time of MHD patients, but also promotes MHD patients to improve their quality of life and help them return to society [7]. At the same time, the expectation of MHD patients is not only to remove metabolic waste from the body and maintain basic survival, but to improve the quality of life [8].

The World Health Organization lists the quality of life as a new health indicator, which can comprehensively evaluate the impact of diseases on life [9]. This study uses the Kidney Disease Quality of Life 36-item(KDQOL ${ }^{\left.\mathrm{TM}_{-}-36\right)}$ to investigate the current status of the quality of life of MHD patients. Among the current measurement scales for the quality of life of MHD patients, KDQOL ${ }^{\mathrm{TM}}-36$ can effectively measure the quality of life and has the advantages of being brief and easy to answer. In order to analyze the influencing factors of the quality of life of MHD patients, 
most researchers found the answer from sociodemographic characteristics, objective physiological and psychological conditions, but ignored the influence of patients' self-feelings of health status on their quality of life. Rather than being a dimension of health, quality of life refers to different dimensions including the self-feelings [6]. Studies have shown that self-feelings of health status has a strong predictive effect on life prognosis [10] and is correlated with mental states such as depression [11], which seriously affects the quality of life. As a consequence, self-feelings of health status is inevitably one of the factors contributing to the quality of life [12].

This study aims to provide a basis for improving the physical, mental health and social activities of MHD patients by analyzing the influencing factors of the quality of life in terms of sociodemographic characteristics, objective physiological conditions and self-feelings of health status.

\section{Materials And Methods Design and Ethics}

This is an analysis of a cross-sectional study that involved outpatients from four hospitals in the coastal area of Zhejiang Province. All procedures were approved by the Institutional Review Board of Affiliated Hospital of Hangzhou Normal University (protocol code:20210009). While collecting raw data, the study was performed in accordance with the provisions of the Helsinki Declaration and subsequent amendments.

\section{Participants and Sample Size}

Patients who had long-term MHD from four hospitals in the coastal area of Zhejiang Province were selected as study participants. Participants were required to meet all the following criteria: subjects diagnosed with ESRD, treated with hemodialysis for at least three months, aged 18 years or older, had no psychiatric or cognitive disorders, were able to read and communicate, were able to complete the questionnaire, were willing to participate in the study and sign informed consent form. The exclusion criteria were as follows: subjects were hospitalized, had serious complications (such as heart failure, severe infection, malignant tumor), with cognitive and behavioral impairment.

\section{Measures}

This study designed the "Questionnaire for Maintenance Hemodialysis Patients" and collected patients information through the questionnaire. The questionnaire consists of 4 parts: the scale of quality of life, sociodemographic characteristics, objective physiological conditions, self-feelings of health status.

\section{The scale of quality of life}

Quality of life was assessed using the KDQOL ${ }^{\mathrm{TM}}-36$ scale. The KDQOL ${ }^{\mathrm{TM}_{-}}-36$ scale is modified and simplified from $\mathrm{KDQOL}^{\mathrm{TM}}$-SF scale, including 12 items derived from general health problems in SF-36 and 24 items derived from symptoms or problems related to kidney disease in $\mathrm{KDQOL}^{\mathrm{TM}}$-SF. This tool consists of five dimensions related to general physical health(items 1-7), general psychological health(items 8-12), kidney disease-related burden(items 13-16), kidney disease-related symptoms(items 17-28) and kidney disease impact(items 29-36). The score range of each dimension is 0-100 points. The higher the score, the better the patient's quality of life [13]. The reliability and validity of the scale has been verified, and the scale can effectively evaluate the quality of life of MHD patients [14].

\section{Sociodemographic characteristics}


Data on age, sex, education level, employment status, medical insurance type, marriage were collected from the MHD patients.

\section{Objective physiological condition}

Data on patient's height, weight, waist circumference, hip circumference, chronic disease were collected from the MHD patients.

\section{Self-feelings of health status}

Data on patient's self-feelings of physical health status, self-feelings of appetite status, self-feelings of sleep quality were collected from the MHD patients.

\section{Statistical Analysis}

All analyses were analyzed with SPSS 20.0 software. Quantitative data was measured by mean and standard deviation, while qualitative data was determined by percentage and proportion. Analysis of variance or t-test was used to analyze the differences in the different dimensions and total scores of kidney disease and quality of life in MHD patients with different characteristics. Multiple linear regression was used to analyze the influencing factors of the quality of life of MHD patients. $P<0.05$ was considered statistically significant for all the analyses.

\section{Result}

\section{Analysis of the sociodemographic characteristics}

A total of $408 \mathrm{MHD}$ patients receiving long-term MHD were included and 251 patients who did not meet the requirements were excluded. After the database was set up, the incomplete data were excluded and the final data included 140 MHD patients(Fig. 1), including 94 males and 46 females. The mean age was $60.91 \pm 14.92$ years. Among the studied MHD patients, 65(46.4\%) patients had an education level of primary school and below, $108(77.1 \%)$ patients were married and only 34(24.3\%) patients were still working. Only two patients had not purchased medical insurance. The sociodemographic characteristics of the patients were presented in Table 1. 
Table 1

Sociodemographic characteristics of MHD patients $(n=140)$

\begin{tabular}{|c|c|c|c|}
\hline variable & & $\mathbf{n}$ & proportion(\%) \\
\hline \multirow[t]{3}{*}{ Age(years) } & $<45$ & 21 & 15.0 \\
\hline & $45-59$ & 39 & 27.9 \\
\hline & $\geq 60$ & 80 & 57.1 \\
\hline \multirow[t]{2}{*}{ Sex } & Male & 94 & 67.1 \\
\hline & Female & 46 & 32.9 \\
\hline \multirow[t]{4}{*}{ Education level } & Primary school and below & 65 & 46.4 \\
\hline & Junior high school & 46 & 32.9 \\
\hline & Senior high school & 18 & 12.9 \\
\hline & College degree and above & 11 & 7.9 \\
\hline \multirow[t]{4}{*}{ Employment status } & On-the-job & 34 & 24.3 \\
\hline & Retired & 71 & 50.7 \\
\hline & Unemployed & 19 & 13.6 \\
\hline & Other & 16 & 11.4 \\
\hline \multirow[t]{5}{*}{ Medical insurance type } & Basic Medical Insurance for urban Employees & 99 & 70.7 \\
\hline & Basic Medical Insurance for urban Residents & 18 & 12.9 \\
\hline & New Rural Cooperative Medical System & 19 & 13.6 \\
\hline & Other & 2 & 1.4 \\
\hline & Not have & 2 & 1.4 \\
\hline \multirow[t]{4}{*}{ Marriage } & Single & 12 & 8.6 \\
\hline & Married & 108 & 77.1 \\
\hline & Divorced & 5 & 3.6 \\
\hline & Widowed & 15 & 10.7 \\
\hline
\end{tabular}

\section{Analysis of the objective physiological condition and self-feelings of health status}

In terms of BMI, 89(63.6\%) patients had normal weight, 21(15.0\%) patients had thin weight and 30(21.5\%) patients were overweight or obese. 133(95.0\%) patients suffered from chronic diseases at the same time, of which hypertension and diabetes were the majority. 16(11.4\%) patients felt that their physical condition was poor, and even 9(6.4\%) patients felt that their physical condition was very poor. $13(9.3 \%)$ patients felt that their appetite was poor and $4(2.9 \%)$ patients felt that their appetite was very poor. 34(24.3\%) patients felt that their sleep quality was poor and even $13(9.3 \%)$ of patients felt that their sleep quality was very poor(Table 2 ). 
Table 2

Objective physiological condition and self-feelings of health status of MHD patients $(n=140)$

\begin{tabular}{|c|c|c|c|}
\hline variable & & $\mathbf{n}$ & proportion(\%) \\
\hline \multirow[t]{4}{*}{ BMI } & Low weight(BMI < 18.5) & 21 & 15.0 \\
\hline & Normal $(18.5 \leq \mathrm{BMI}<24)$ & 89 & 63.6 \\
\hline & 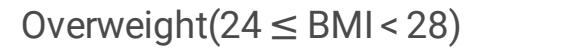 & 26 & 18.6 \\
\hline & Obesity(BMI $\geq 28)$ & 4 & 2.9 \\
\hline \multirow[t]{2}{*}{ Waist to hip ratio } & Normal & 81 & 57.9 \\
\hline & Obesity(Men $\geq 0.9$ Female $\geq 0.8$ ) & 59 & 42.1 \\
\hline \multirow[t]{7}{*}{ Chronic disease } & No & 7 & 5.0 \\
\hline & Hypertension & 125 & 89.3 \\
\hline & Diabetes & 56 & 40.0 \\
\hline & Cardiovascular disease & 37 & 26.4 \\
\hline & Fatty liver & 16 & 11.4 \\
\hline & Cancer & 5 & 3.6 \\
\hline & Other & 35 & 25.0 \\
\hline \multirow[t]{5}{*}{ Self-feelings of physical health status } & Very good & 27 & 19.3 \\
\hline & Good & 57 & 40.7 \\
\hline & Fair & 31 & 22.1 \\
\hline & Poor & 16 & 11.4 \\
\hline & Very poor & 9 & 6.4 \\
\hline \multirow[t]{5}{*}{ Self-feelings of appetite status } & Very good & 34 & 24.3 \\
\hline & Good & 63 & 45.0 \\
\hline & Fair & 26 & 18.6 \\
\hline & Poor & 13 & 9.3 \\
\hline & Very poor & 4 & 2.9 \\
\hline \multirow[t]{5}{*}{ Self-feelings of sleep quality } & Very good & 22 & 15.7 \\
\hline & Good & 33 & 23.6 \\
\hline & Fair & 38 & 27.1 \\
\hline & Poor & 34 & 24.3 \\
\hline & Very poor & 13 & 9.3 \\
\hline
\end{tabular}

\section{Analysis of the quality of life}


The average score of Kidney disease and Quality of life was $264.50 \pm 41.92$, of which the average score of general physical health was $42.89 \pm 9.86$, the average score of general psychological health was $46.72 \pm 7.12$, the average score of kidney disease-related symptoms was $77.95 \pm 10.82$, the average score of kidney disease impact was $64.98 \pm 12.03$ and the average score of kidney disease-related burden was $32.01 \pm 19.35$. Among them, 131(93.6\%) patients suffered from exhaustion, followed by itchy skin(85.7\%), dry skin(78.6\%) and cramping(71.4\%)(Fig. 2). In terms of kidney disease impact, $135(96.4 \%)$ patients were troubled with the restriction of drinking fluids, followed by dietary restrictions(95.0\%), stress or anxiety(92.9\%)(Fig. 3).

In terms of general physical health and kidney disease-related burden, the scores in females were lower than that of males $(P<0.05)$. Patients in different age groups had different scores in general physical health, kidney diseaserelated symptoms and kidney disease impact $(P<0.05)$. Patients with different education levels had different scores in general physical health and kidney disease-related symptoms $(P<0.05)$. Patients with different employment status had different scores in kidney disease-related symptoms, kidney disease impact and kidney disease-related burden $(P<0.05)$. Patients with different marital status had different scores in general physical health, general psychological health and kidney disease-related symptoms $(P<0.05)$. Patients with different self-feelings of physical health status had different scores in each dimension. Patients with different self-feelings of appetite status and different self-feelings of sleep quality levels had different scores in each dimension except kidney disease impact. (Table 3). 
Table 3

The scores of Kidney disease and quality of life in each dimension $( \pm s)$

\begin{tabular}{|c|c|c|c|c|c|c|c|c|}
\hline variable & & $n$ & $\begin{array}{l}\text { General } \\
\text { physical } \\
\text { health }\end{array}$ & $\begin{array}{l}\text { General } \\
\text { psychological } \\
\text { health }\end{array}$ & $\begin{array}{l}\text { Kidney } \\
\text { disease- } \\
\text { related } \\
\text { symptoms }\end{array}$ & $\begin{array}{l}\text { Kidney } \\
\text { disease } \\
\text { impact }\end{array}$ & $\begin{array}{l}\text { Kidney } \\
\text { disease- } \\
\text { related } \\
\text { burden }\end{array}$ & $\begin{array}{l}\text { Total } \\
\text { score }\end{array}$ \\
\hline \multirow[t]{4}{*}{ Sex } & Men & 94 & $\begin{array}{l}44.37 \pm \\
8.51\end{array}$ & $47.49 \pm 7.32$ & $\begin{array}{l}79.06 \pm \\
10.05\end{array}$ & $\begin{array}{l}64.86 \\
\pm 11.15\end{array}$ & $\begin{array}{l}34.31 \pm \\
20.37\end{array}$ & $\begin{array}{l}270.08 \\
\pm 39.25\end{array}$ \\
\hline & Female & 46 & $\begin{array}{l}39.89 \pm \\
11.71\end{array}$ & $45.15 \pm 7.45$ & $\begin{array}{l}75.68 \pm \\
12.05\end{array}$ & $\begin{array}{l}65.22 \\
\pm 13.78\end{array}$ & $\begin{array}{l}27.31 \pm \\
16.32\end{array}$ & $\begin{array}{l}253.09 \\
\pm 45.22\end{array}$ \\
\hline & $t$ & & 2.313 & 1.770 & 1.747 & -0.164 & 2.032 & 2.287 \\
\hline & $P$ & & $0.024 *$ & 0.079 & 0.083 & 0.870 & $0.044^{\star}$ & $0.024 *$ \\
\hline \multirow{5}{*}{$\begin{array}{l}\text { Age } \\
\text { (years) }\end{array}$} & $<45$ & 21 & $\begin{array}{l}48.98 \pm \\
5.86\end{array}$ & $46.14 \pm 7.20$ & $\begin{array}{l}84.72 \pm \\
7.64\end{array}$ & $\begin{array}{l}57.74 \\
\pm 9.66\end{array}$ & $\begin{array}{l}26.79 \pm \\
20.27\end{array}$ & $\begin{array}{l}264.38 \\
\pm 34.89\end{array}$ \\
\hline & $45-59$ & 39 & $\begin{array}{l}41.82 \pm \\
9.16\end{array}$ & $47.52 \pm 6.23$ & $\begin{array}{l}79.49 \pm \\
9.47\end{array}$ & $\begin{array}{l}61.22 \\
\pm 13.43\end{array}$ & $\begin{array}{l}28.21 \pm \\
18.30\end{array}$ & $\begin{array}{l}258.25 \\
\pm 43.76\end{array}$ \\
\hline & $\geq 60$ & 80 & $\begin{array}{l}41.82 \pm \\
10.51\end{array}$ & $46.48 \pm 8.04$ & $\begin{array}{l}75.42 \pm \\
11.33\end{array}$ & $\begin{array}{l}68.71 \\
\pm 10.45\end{array}$ & $\begin{array}{l}35.23 \pm \\
19.21\end{array}$ & $\begin{array}{l}267.58 \\
\pm 42.82\end{array}$ \\
\hline & $F$ & & 4.980 & 0.327 & 7.307 & 10.931 & 2.693 & 0.646 \\
\hline & $P$ & & $0.008 *$ & 0.722 & $0.001 *$ & <. $001 *$ & 0.071 & 0.526 \\
\hline \multirow[t]{6}{*}{$\begin{array}{l}\text { Education } \\
\text { levels }\end{array}$} & $\begin{array}{l}\text { Primary } \\
\text { school and } \\
\text { below }\end{array}$ & 65 & $\begin{array}{l}40.32 \pm \\
10.44\end{array}$ & $46.15 \pm 8.07$ & $\begin{array}{l}74.61 \pm \\
11.12\end{array}$ & $\begin{array}{l}66.93 \\
\pm 11.98\end{array}$ & $\begin{array}{l}28.75 \pm \\
16.79\end{array}$ & $\begin{array}{l}256.65 \\
\pm 41.73\end{array}$ \\
\hline & $\begin{array}{l}\text { Junior high } \\
\text { school }\end{array}$ & 46 & $\begin{array}{l}44.88 \pm \\
9.29\end{array}$ & $46.06 \pm 6.77$ & $\begin{array}{l}79.85 \pm \\
10.00\end{array}$ & $\begin{array}{l}63.39 \\
\pm 13.17\end{array}$ & $\begin{array}{l}32.74 \pm \\
21.78\end{array}$ & $\begin{array}{l}266.91 \\
\pm 45.13\end{array}$ \\
\hline & $\begin{array}{l}\text { Senior high } \\
\text { school }\end{array}$ & 18 & $\begin{array}{l}43.65 \pm \\
8.38\end{array}$ & $47.97 \pm 7.20$ & $\begin{array}{l}80.32 \pm \\
9.51\end{array}$ & $\begin{array}{l}65.11 \\
\pm 9.60\end{array}$ & $\begin{array}{l}37.15 \pm \\
17.08\end{array}$ & $\begin{array}{l}274.20 \\
\pm 32.01\end{array}$ \\
\hline & $\begin{array}{l}\text { College } \\
\text { degree and } \\
\text { above }\end{array}$ & 11 & $\begin{array}{l}48.58 \pm \\
6.98\end{array}$ & $50.78 \pm 4.39$ & $\begin{array}{l}85.80 \pm \\
8.11\end{array}$ & $\begin{array}{l}59.95 \\
\pm 9.56\end{array}$ & $\begin{array}{l}39.77 \pm \\
24.25\end{array}$ & $\begin{array}{l}284.88 \\
\pm 36.35\end{array}$ \\
\hline & $F$ & & 3.543 & 1.539 & 5.173 & 1.495 & 1.674 & 2.043 \\
\hline & $P$ & & $0.016 *$ & 0.207 & $0.002^{*}$ & 0.219 & 0.175 & 0.111 \\
\hline \multirow[t]{4}{*}{$\begin{array}{l}\text { Employment } \\
\text { status }\end{array}$} & On-the-job & 34 & $\begin{array}{l}45.37 \pm \\
6.56\end{array}$ & $47.45 \pm 6.74$ & $\begin{array}{l}82.78 \pm \\
7.82\end{array}$ & $\begin{array}{l}59.65 \\
\pm 11.42\end{array}$ & $\begin{array}{l}26.84 \pm \\
18.56\end{array}$ & $\begin{array}{l}262.09 \\
\pm 34.74\end{array}$ \\
\hline & Retired & 71 & $\begin{array}{l}41.47 \pm \\
10.64\end{array}$ & $47.34 \pm 7.20$ & $\begin{array}{l}77.02 \pm \\
10.90\end{array}$ & $\begin{array}{l}66.86 \\
\pm 11.85\end{array}$ & $\begin{array}{l}37.94 \pm \\
19.05\end{array}$ & $\begin{array}{l}270.54 \\
\pm 44.43\end{array}$ \\
\hline & Unemployed & 19 & $\begin{array}{l}43.39 \pm \\
9.77\end{array}$ & $44.25 \pm 5.49$ & $\begin{array}{l}78.29 \pm \\
10.09\end{array}$ & $\begin{array}{l}62.34 \\
\pm 11.90\end{array}$ & $\begin{array}{l}22.04 \\
15.36\end{array}$ & $\begin{array}{l}250.30 \\
\pm 37.10\end{array}$ \\
\hline & Other & 16 & $\begin{array}{l}43.36 \pm \\
11.84\end{array}$ & $45.35 \pm 10.94$ & $\begin{array}{l}71.35 \pm \\
13.03\end{array}$ & $\begin{array}{l}71.10 \\
\pm 13.03\end{array}$ & $\begin{array}{l}28.52 \pm \\
19.23\end{array}$ & $\begin{array}{l}259.68 \\
\pm 47.96\end{array}$ \\
\hline
\end{tabular}




\begin{tabular}{|c|c|c|c|c|c|c|c|c|}
\hline \multirow[t]{3}{*}{ variable } & & $n$ & $\begin{array}{l}\text { General } \\
\text { physical } \\
\text { health }\end{array}$ & $\begin{array}{l}\text { General } \\
\text { psychological } \\
\text { health }\end{array}$ & $\begin{array}{l}\text { Kidney } \\
\text { disease- } \\
\text { related } \\
\text { symptoms }\end{array}$ & $\begin{array}{l}\text { Kidney } \\
\text { disease } \\
\text { impact }\end{array}$ & $\begin{array}{l}\text { Kidney } \\
\text { disease- } \\
\text { related } \\
\text { burden }\end{array}$ & $\begin{array}{l}\text { Total } \\
\text { score }\end{array}$ \\
\hline & $F$ & & 1.239 & 1.163 & 4.782 & 4.859 & 5.344 & 1.335 \\
\hline & $P$ & & 0.298 & 0.326 & $0.003^{*}$ & $0.003^{*}$ & $0.002^{*}$ & 0.266 \\
\hline \multirow[t]{7}{*}{$\begin{array}{l}\text { Medical } \\
\text { insurance } \\
\text { types }\end{array}$} & $\begin{array}{l}\text { Basic } \\
\text { Medical } \\
\text { Insurance } \\
\text { for urban } \\
\text { Employees }\end{array}$ & 99 & $\begin{array}{l}43.20 \pm \\
9.73\end{array}$ & $46.98 \pm 7.04$ & $\begin{array}{l}79.19 \pm \\
10.76\end{array}$ & $\begin{array}{l}64.27 \\
\pm 12.12\end{array}$ & $\begin{array}{l}33.78 \pm \\
19.52\end{array}$ & $\begin{array}{l}267.41 \\
\pm 42.16\end{array}$ \\
\hline & $\begin{array}{l}\text { Basic } \\
\text { Medical } \\
\text { Insurance } \\
\text { for urban } \\
\text { Residents }\end{array}$ & 18 & $\begin{array}{l}38.44 \pm \\
11.50\end{array}$ & $45.51 \pm 7.79$ & $\begin{array}{l}72.80 \pm \\
12.97\end{array}$ & $\begin{array}{l}65.45 \\
\pm 14.36\end{array}$ & $\begin{array}{l}26.74 \pm \\
19.27\end{array}$ & $\begin{array}{l}248.93 \\
\pm 44.57\end{array}$ \\
\hline & $\begin{array}{l}\text { New Rural } \\
\text { Cooperative } \\
\text { Medical } \\
\text { System }\end{array}$ & 19 & $\begin{array}{l}45.56 \pm \\
8.50\end{array}$ & $44.83 \pm 8.77$ & $\begin{array}{l}76.21 \pm \\
8.48\end{array}$ & $\begin{array}{l}68.09 \\
\pm 10.29\end{array}$ & $\begin{array}{l}27.30 \pm \\
18.31\end{array}$ & $\begin{array}{l}261.62 \\
\pm 39.08\end{array}$ \\
\hline & Other & 2 & $\begin{array}{l}36.74 \pm \\
1.15\end{array}$ & $53.35 \pm 3.01$ & $\begin{array}{l}79.17 \pm \\
8.84\end{array}$ & $\begin{array}{l}67.19 \\
\pm 6.63\end{array}$ & $\begin{array}{l}21.88 \pm \\
22.08\end{array}$ & $\begin{array}{l}258.32 \\
\pm 39.43\end{array}$ \\
\hline & Not have & 2 & $\begin{array}{l}48.57 \pm \\
6.53\end{array}$ & $56.16 \pm 1.61$ & $\begin{array}{l}78.13 \pm \\
1.48\end{array}$ & $\begin{array}{l}64.07 \\
\pm 2.21\end{array}$ & $\begin{array}{l}46.88 \pm \\
4.42\end{array}$ & $\begin{array}{l}293.79 \\
\pm 8.87\end{array}$ \\
\hline & $F$ & & 1.684 & 1.702 & 1.494 & 0.424 & 1.263 & 1.018 \\
\hline & $P$ & & 0.157 & 0.153 & 0.208 & 0.791 & 0.288 & 0.400 \\
\hline \multirow[t]{6}{*}{ Marriage } & Single & 12 & $\begin{array}{l}49.42 \pm \\
7.51\end{array}$ & $47.17 \pm 5.51$ & $\begin{array}{l}84.38 \pm \\
8.54\end{array}$ & $\begin{array}{l}63.28 \\
\pm 11.08\end{array}$ & $\begin{array}{l}31.77 \pm \\
19.30\end{array}$ & $\begin{array}{l}276.02 \\
\pm 37.87\end{array}$ \\
\hline & Married & 108 & $\begin{array}{l}43.55 \pm \\
8.81\end{array}$ & $47.56 \pm 7.31$ & $\begin{array}{l}78.53 \pm \\
9.87\end{array}$ & $\begin{array}{l}65.28 \\
\pm 12.19\end{array}$ & $\begin{array}{l}33.62 \pm \\
19.10\end{array}$ & $\begin{array}{l}268.47 \\
\pm 38.14\end{array}$ \\
\hline & Divorced & 5 & $\begin{array}{l}35.38 \pm \\
11.27\end{array}$ & $40.76 \pm 8.34$ & $\begin{array}{l}62.92 \pm \\
12.09\end{array}$ & $\begin{array}{l}60.00 \\
\pm 13.51\end{array}$ & $\begin{array}{l}21.25 \pm \\
20.54\end{array}$ & $\begin{array}{l}220.31 \\
\pm 55.92\end{array}$ \\
\hline & Widowed & 15 & $\begin{array}{l}35.48 \pm \\
13.15\end{array}$ & $42.32 \pm 7.43$ & $\begin{array}{l}73.61 \pm \\
13.42\end{array}$ & $\begin{array}{l}65.84 \\
\pm 11.78\end{array}$ & $\begin{array}{l}24.17 \pm \\
19.75\end{array}$ & $\begin{array}{l}241.41 \\
\pm 52.97\end{array}$ \\
\hline & $F$ & & 6.363 & 3.486 & 6.150 & 0.408 & 1.608 & 4.276 \\
\hline & $P$ & & $<0.001^{*}$ & $0.018^{*}$ & $0.001 *$ & 0.748 & 0.191 & $0.006^{*}$ \\
\hline \multirow{4}{*}{$\begin{array}{l}\text { Self-feelings } \\
\text { of physical } \\
\text { health }\end{array}$} & Good & 84 & $\begin{array}{l}46.84 \pm \\
7.60\end{array}$ & $48.85 \pm 6.16$ & $\begin{array}{l}81.65 \pm \\
8.89\end{array}$ & $\begin{array}{l}65.78 \\
\pm 11.53\end{array}$ & $\begin{array}{l}36.16 \pm \\
19.67\end{array}$ & $\begin{array}{l}279.19 \\
\pm 34.55\end{array}$ \\
\hline & Fair & 31 & $\begin{array}{l}41.33 \pm \\
8.83\end{array}$ & $45.42 \pm 7.94$ & $\begin{array}{l}75.34 \pm \\
9.00\end{array}$ & $\begin{array}{l}67.04 \\
\pm 11.66\end{array}$ & $\begin{array}{l}29.64 \pm \\
16.85\end{array}$ & $\begin{array}{l}258.76 \\
\pm 34.13\end{array}$ \\
\hline & Poor & 25 & $\begin{array}{l}31.57 \pm \\
8.65\end{array}$ & $41.17 \pm 7.67$ & $\begin{array}{l}68.75 \pm \\
12.95\end{array}$ & $\begin{array}{l}59.75 \\
\pm 13.11\end{array}$ & $\begin{array}{l}21.00 \pm \\
16.82\end{array}$ & $\begin{array}{l}222.24 \\
\pm 44.00\end{array}$ \\
\hline & $F$ & & 35.231 & 12.783 & 18.602 & 3.090 & 6.721 & 24.215 \\
\hline
\end{tabular}




\begin{tabular}{|c|c|c|c|c|c|c|c|c|}
\hline \multirow[t]{2}{*}{ variable } & & $n$ & $\begin{array}{l}\text { General } \\
\text { physical } \\
\text { health }\end{array}$ & $\begin{array}{l}\text { General } \\
\text { psychological } \\
\text { health }\end{array}$ & $\begin{array}{l}\text { Kidney } \\
\text { disease- } \\
\text { related } \\
\text { symptoms }\end{array}$ & $\begin{array}{l}\text { Kidney } \\
\text { disease } \\
\text { impact }\end{array}$ & $\begin{array}{l}\text { Kidney } \\
\text { disease- } \\
\text { related } \\
\text { burden }\end{array}$ & $\begin{array}{l}\text { Total } \\
\text { score }\end{array}$ \\
\hline & $P$ & & $<0.001 *$ & $0.001^{*}$ & $<0.001 *$ & $0.049 *$ & $0.002^{\star}$ & $\stackrel{<}{0.001 *}$ \\
\hline \multirow{5}{*}{$\begin{array}{l}\text { Self-feelings } \\
\text { of appetite } \\
\text { status }\end{array}$} & Good & 97 & $\begin{array}{l}46.15 \pm \\
7.73\end{array}$ & $48.03 \pm 7.06$ & $\begin{array}{l}81.36 \pm \\
8.81\end{array}$ & $\begin{array}{l}64.98 \\
\pm 11.31\end{array}$ & $\begin{array}{l}34.66 \pm \\
18.62\end{array}$ & $\begin{array}{l}275.11 \\
\pm 34.99\end{array}$ \\
\hline & Fair & 26 & $\begin{array}{l}38.57 \pm \\
11.10\end{array}$ & $45.76 \pm 6.50$ & $\begin{array}{l}71.80 \pm \\
12.08\end{array}$ & $\begin{array}{l}67.43 \\
\pm 11.22\end{array}$ & $\begin{array}{l}30.77 \pm \\
22.22\end{array}$ & $\begin{array}{l}254.32 \\
\pm 47.70\end{array}$ \\
\hline & Poor & 17 & $\begin{array}{l}30.96 \pm \\
6.94\end{array}$ & $40.69 \pm 7.89$ & $\begin{array}{l}67.89 \pm \\
9.17\end{array}$ & $\begin{array}{l}61.22 \\
\pm 16.35\end{array}$ & $\begin{array}{l}18.75 \pm \\
13.07\end{array}$ & $\begin{array}{l}219.51 \\
\pm 36.96\end{array}$ \\
\hline & $F$ & & 28.097 & 8.104 & 21.084 & 1.379 & 5.260 & 16.763 \\
\hline & $P$ & & $<0.001^{*}$ & $<0.001^{*}$ & $<0.001^{*}$ & 0.255 & $0.006^{*}$ & $<.001 *$ \\
\hline \multirow{5}{*}{$\begin{array}{l}\text { Self-feelings } \\
\text { of sleep } \\
\text { quality }\end{array}$} & Good & 55 & $\begin{array}{l}47.50 \pm \\
6.71\end{array}$ & $49.32 \pm 6.22$ & $\begin{array}{l}82.54 \pm \\
8.99\end{array}$ & $\begin{array}{l}67.05 \\
\pm 11.20\end{array}$ & $\begin{array}{l}36.59 \pm \\
16.77\end{array}$ & $\begin{array}{l}283.00 \\
\pm 32.13\end{array}$ \\
\hline & Fair & 38 & $\begin{array}{l}42.36 \pm \\
10.98\end{array}$ & $45.99 \pm 7.58$ & $\begin{array}{l}76.97 \pm \\
10.51\end{array}$ & $\begin{array}{l}63.08 \\
\pm 13.09\end{array}$ & $\begin{array}{l}31.74 \pm \\
24.11\end{array}$ & $\begin{array}{l}260.14 \\
\pm 46.89\end{array}$ \\
\hline & Poor & 47 & $\begin{array}{l}37.94 \pm \\
9.65\end{array}$ & $44.27 \pm 7.75$ & $\begin{array}{l}73.36 \pm \\
11.07\end{array}$ & $\begin{array}{l}64.10 \\
\pm 11.96\end{array}$ & $\begin{array}{l}26.86 \pm \\
16.79\end{array}$ & $\begin{array}{l}246.37 \\
\pm 39.62\end{array}$ \\
\hline & $F$ & & 14.297 & 6.615 & 10.617 & 1.423 & 3.314 & 11.448 \\
\hline & $P$ & & $<0.001^{*}$ & $0.002^{*}$ & $<0.001^{*}$ & 0.245 & $0.039 *$ & $<.001 *$ \\
\hline
\end{tabular}

\section{Influencing factors of MHD patients' quality of life}

To determine the relationship of quality of life with sociodemographic characteristics, objective physiological condition and self-feelings of health status, multiple linear regression analysis was used. The results showed that self-feelings of physical health status, self-feelings of appetite status and self-feelings of sleep quality were included in the regression model. The quality of life of the patients with bad self-feelings of physical health status, bad self-feelings of appetite status and bad feelings of sleep quality was worse than those patients with good selffeelings $(P<0.05)$ (Table 4$)$. 
Table 4

Multiple linear regression analysis of quality of life

\begin{tabular}{|c|c|c|c|c|c|}
\hline variable & $B$ & $S_{x}^{-}$ & $\boldsymbol{\beta}$ & $t$ & $P$ \\
\hline constant & 283.508 & 11.833 & & 23.959 & $<0.001$ \\
\hline Sex(Control group = Female) & 9.068 & 6.827 & 0.102 & 1.328 & 0.186 \\
\hline \multicolumn{6}{|c|}{ Marriage(Control group = Single ) } \\
\hline Married & -1.914 & 10.859 & -0.019 & -0.176 & 0.860 \\
\hline Divorced & -11.337 & 19.560 & -0.050 & -0.580 & 0.563 \\
\hline Widowed & -6.158 & 14.570 & -0.046 & -0.423 & 0.673 \\
\hline \multicolumn{6}{|c|}{ Self-feelings of physical health status(Control group = Good) } \\
\hline Fair & -8.207 & 8.122 & -0.082 & -1.010 & 0.314 \\
\hline Poor & -38.213 & 9.622 & -0.350 & -3.971 & $<0.001^{*}$ \\
\hline \multicolumn{6}{|c|}{ Self-feelings of appetite status(Control group = Good) } \\
\hline Fair & -3.103 & 8.306 & -0.029 & -0.374 & 0.709 \\
\hline Poor & -28.300 & 10.573 & -0.221 & -2.677 & $0.008^{*}$ \\
\hline \multicolumn{6}{|c|}{ Self-feelings of sleep quality(Control group = Good) } \\
\hline Fair & -14.571 & 7.967 & -0.155 & -1.829 & 0.070 \\
\hline Poor & -17.718 & 8.281 & -0.200 & -2.140 & $0.034 *$ \\
\hline
\end{tabular}

\section{Discussion}

\section{Health status of MHD patients}

This study found that most MHD patients had normal weight and waist to hip ratio, which reflects that most MHD patients have a good body shape. The results are similar to those of related studies [15]. Also this study found that most MHD patients suffer from other chronic diseases at the same time, among which hypertension and diabetes were the majority. Previous studies also have shown that the underlying diseases in MHD patients were mostly diabetic nephropathy and hypertensive nephropathy $[16,17]$ and chronic diseases such as hypertension and diabetes may impair the physical function of MHD patients and reduce their quality of life [18]. This study comprehensively analyzed the self-feelings of health status of MHD patients through self-feelings of physical health status, self-feelings of appetite status and self-feelings of sleep quality. However, previous studies only conducted research from a single aspect such as diet status or sleep disorders. In this study, MHD patients had better selffeelings of physical health status, self-feelings of appetite status and self-feelings of sleep quality while previous studies have shown that MHD patients have poor diet status [19] and a higher prevalence of sleep disorders [20]. The possible reason was that the subjects of this study were all from the coastal areas of Zhejiang Province. According to the national GDP ranking, the economic development level and people's living standards were relatively 
high in Zhejiang Province. Therefore, there were more choices in diet according to the preferences of MHD patients, which was possible improve the MHD patient's appetite status. In addition, in terms of sleep conditions, previous studies have mostly used objective indicators to evaluate patients' sleep quality and poor sleep quality had a broader definition. However, this study used subjective indicators to evaluate MHD patients' self-feelings of sleep quality. Patients often think that poor sleep quality is only due to insufficient sleep duration or frequent awakenings.

\section{The quality of life of MHD patients}

Among the scores on the dimensions of kidney disease and quality of life in MHD patients in this study, the kidney disease-related burden dimension had the lowest score and the kidney disease-related symptoms dimension had the highest score. This study showed that MHD patients were mainly troubled by exhaustion, itchy skin, dry skin and cramps, which was similar to the results of related studies [21]. Relevant studies have shown that fatigue was one of the most common symptoms of MHD patients, with an incidence of $60 \%-97 \%[22,23]$ and $46 \%$ of MHD patients suffered from moderate or severe skin itching [24]. These symptoms seriously interfered with the normal life of MHD patients and became a common reason for MHD patients' unwillingness to follow regular hemodialysis and were also an important factor leading to poor prognosis of MHD patients. This suggested that medical staff should pay more attention to the symptoms of MHD patients, such as fatigue and skin itching. Also medical staff should improve the clinical compliance of MHD patients by relieving such symptoms, so as to improve the quality of life of MHD patients. On the other hand, MHD patients were more troubled with fluid restrictions, dietary restrictions and stress or anxiety. MHD patients need to appropriately limit water intake, reduce protein intake, reduce phosphorus intake, monitor sodium intake, potassium intake and limit fixed acid. Due to strict dietary restrictions and dialysisrelated intestinal reactions, more than $30 \%$ of MHD patients will have anorexia symptoms [21]. In addition, studies have shown that MHD patients need to spend a lot of treatment costs for long-term treatment. Long-term pain and huge economic burden often make patients face great pressure and doubt the value of their own existence, so as to face life negatively. At the same time, although MHD can prolong the survival time of patients, but it can not improve the renal function of patients, the shadow of death is always around the patients, resulting in fear, anxiety and helplessness. The physical and mental negative impact seriously interferes with the normal life of MHD patients [25, 26]. This suggests that medical staff and family members need to pay more attention to the emotional changes of MHD patients, and relieve patients' unhealthy emotions through correct guidance.

\section{Influencing factors of the quality of life of MHD patients}

This study found that male patients have better physical health, a smaller burden of kidney disease and a higher quality of life. This may be related to the different physical characteristics of male and female. In addition to the development of the disease itself, women's physiological function declines more significantly as the disease occurs and develops. However, previous studies showed that in the process of facing the disease, the mental state of men is often better than that of women. But this study found no differences in general psychological health scores between male patients and female patients. This may be related to the good level of medical care and the good humanistic care of medical staff. So that medical staff need pay more attention to female patients' physical conditions.

In this study, patients under 45 years old had the best physical condition and the least trouble with kidney diseaserelated symptoms. In addition to the effects of the disease itself, as the age increases, the functions of various organs in MHD patients will also decline. The various complications and decline in abilities brought about by aging directly lead to the decline of physical conditions [27]. In terms of the kidney disease impact, the results showed that the patients over 60 years old were less affected, possibly because most of these patients have retired and there are 
not too many tasks to be completed in time in daily life, so the impact is relatively little. For patients under 45 years old, their employment pressure is high and there are many household chores to deal with, so the impact of kidney disease on daily life is relatively greater.

In terms of educational level, the higher the educational level, the better physical condition and the less troubled with kidney disease-related symptoms. Patients with better education have more ways to obtain disease-related information, can be more proactive in understanding disease-related knowledge and better cooperate with medical staff in treatment. At the same time, patients with a high level of education pay more attention to themselves and are easy to accept new information and knowledge [28]. These suggest that medical staff need to provide more health education knowledge to improve the health literacy of MHD patients, thereby improving the quality of life.

Employed patients were less troubled with kidney disease-related symptoms. However, retired patients had less burden of kidney disease. Previous studies have found that employment could improve patients' sense of selfesteem and promote their social communication and the physical and social function scores of employed patients are relatively high $[29,30]$. So their psychological status and social support were better and they were less troubled with symptoms. This suggests that MHD patients need to actively contact with society and friends, avoid being away from society and need to relieve emotional stress in time. For retired patients, their daily material and spiritual needs are little, their economic income is sufficient to meet their daily needs and their social welfare benefits are relatively good, so their kidney disease-related burden is the smallest.

Unmarried patients had better physical conditions, were less troubled with symptoms of kidney disease and had a higher quality of life. The possible reason was that unmarried patients were younger than other patients and had relatively better physical functions. Previous studies have shown that most married patients cannot perform their duties of supporting their parents and raising children normally and they also need to rely on the care of others, which causes their burden to be heavier [31]. This indirectly causes married patients to become more troubled with the symptoms of kidney disease, which leads to a decline in their quality of life. However, this study showed that married patients had better psychological health. The possible reason was that married patients are more able to obtain family support and relieve negative emotions.

This study found that self-feelings of health status had a greater impact on the quality of life of MHD patients. Patients with good self-feelings of physical health status had better physical and psychological health, were less troubled with symptoms of kidney disease, had less kidney disease impact and less burden of kidney disease and had better quality of life. The possible reason is that patients with good self-feelings of physical health status have a better mental state and a more positive attitude towards disease and they are more confident to overcome disease-related symptoms, while patients with bad self-feelings of physical health status have more depression in their lives. Patients with good self-feelings of appetite status had better physical and psychological health, were least troubled with kidney disease symptoms and had a better quality of life. Relevant studies have shown that decreased appetite will increase protein energy consumption in MHD patients, resulting in decreased nutritional status of MHD patients [32]. This makes the symptoms of kidney disease aggravate the trouble for MHD patients, which indirectly causes the quality of life of MHD patients to decline. However, patients with good self-feelings of appetite status can feel more delicious food when eating, thereby contributing to the balanced intake of nutrients, reducing disease-related symptoms and improving the quality of life. Patients with good self-feelings of sleep quality have better physical and psychological health, were less troubled with kidney disease symptoms, had less burden of kidney disease and had better quality of life. Studies have shown that patients with poor self-feelings of sleep quality were more likely to experience fatigue and excessive sleepiness during the day, which disrupted normal 
circadian rhythms [33]. In addition, poor self-feelings of sleep quality in MHD patients will aggravate their negative emotions such as anxiety, depression, irritability and cause listlessness, dizziness, drowsiness, fatigue, loss of appetite and other symptoms during the day and they do not have enough energy and physical strength to devote themselves to normal life [33]. Poor self-feelings of sleep quality and symptom distress affect each other and seriously affect the quality of life of MHD patients.

After multiple linear regression analysis, self-feelings of physical health status, self-feelings of appetite status and self-feelings of sleep quality were the influencing factors of quality of life. Patients with poor self-feelings of physical health status, poor self-feelings of appetite status and poor self-feelings of sleep quality had worse quality of life. This suggests that medical staff should pay more attention to the self-feelings of patients. The most intuitive manifestation of a patient's health status is his self-feelings of health. It is necessary to strengthen the dietary guidance of MHD patients. At present, medical staff often only pay attention to what MHD patients can or cannot eat, but ignore their diet preference and appetite [19], which may cause MHD patients' poor dietary compliance to doctors' guidance. Medical staff can provide targeted dietary guidance programs based on the main factors affecting the diet of MHD patients and the patient's eating habits, so as to improve the quality of life of MHD patients. At the same time, medical staff should pay more attention to the sleep status and daytime mental status of MHD patients. For patients with poor sleep, it is necessary to guide them to improve their sleep quality by improving their sleeping environment, listening to music, soaking their feet or reducing the use of electronic devices before going to bed.

The limitation of this study is that the samples in this study are all from the coastal areas of Zhejiang Province, which can only represent the situation of the more developed areas in China. However, further research should be conducted on the underdeveloped areas in western China.

\section{Conclusion}

This study evaluated the quality of life of MHD patients in coastal areas of Zhejiang Province through questionnaire survey and explored the influencing factors of quality of life. The results showed that MHD patients with different sociodemographic characteristics and self-feelings of health status had different scores in different dimensions. Self-feelings of physical health status, self-feelings of appetite status and self-feelings of sleep quality are the main influencing factors of MHD patients' quality of life. Therefore, medical staff should pay full attention to the selffeelings of health status of MHD patients, improve their appetite through targeted dietary guidance and improve their sleep quality by providing sleep tips. Also medical staff need to provide psychological guidance and ease MHD patients' negative emotions, so as to reduce their fear of disease and improve their self-feelings of health status and improve the quality of life.

\section{Abbreviations}

MHD: Maintenance hemodialysis; ESRD: End-stage renal disease; KDQOL ${ }^{\mathrm{TM}}-36$ : the Kidney Disease Quality of Life 36-item

\section{Declarations}

Acknowledgments: The author is very grateful to the survey team for providing support for the recruitment of participants, questionnaire surveys and information collection. The author thanks all members who contributed to this work. 
Author Contributions: M.G., F.M.: research idea, study design, statistical analysis and drafting of the manuscript. M.G., Z.J., Y.H.: data collection, analysis and interpretation. Q.G., Y.M.: revision and approval of the manuscript. All authors read and approved the final manuscript.

Funding: This research was funded by Publicity Department, National Health and Family Planning Commission, grant number 4125F40214090.

Ethical Approval and Consent to participate: The study was conducted according to the guidelines of the Declaration of Helsinki and approved by the Institutional Review Board of Affiliated Hospital of Hangzhou Normal University (20210009, January 2021). Informed consent was obtained from all subjects involved in the study. Written informed consent has been obtained from the patients to publish this paper.

Consent for publication】All presentations of case reports have consent for publication.

Availability of data and materials: The datasets used and/or analysed during the current study are available from the corresponding author on reasonable request.

Conflicts of Interest: The authors declare that they have no competing interests.

\section{References}

1. Teles F, Azevedo VF, Miranda CT, Miranda MP, Teixeira Mdo C, Elias RM. Depression in hemodialysis patients: the role of dialysis shift. Clinics (Sao Paulo). 2014 Mar;69(3):198-202.

2. Weisbord SD, Mor MK, Sevick MA, Shields AM, Rollman BL, Palevsky PM, Arnold RM, Green JA, Fine MJ. Associations of depressive symptoms and pain with dialysis adherence, health resource utilization, and mortality in patients receiving chronic hemodialysis. Clin J Am Soc Nephrol. 2014 Sep 5;9(9):1594-602.

3. Gu B, Qiao Q, Ma J. Epidemiological investigation of end stage renal disease patients receiving hemodialysis in Jing'an District in Shanghai.Shanghai Medical Journal,2015,38(1):846-850.

4. Wu H, Wen XX, Li Y, Lei H. Analysis of Disease Uncertainty and Influencing Factors in Maintenance Hemodialysis Patients.Journal of Qilu Nursing,2020,26(13):17-21.

5. Wan EY, Chen JY, Choi EP, Wong CK, Chan AK, Chan KH, Lam CL. Patterns of health-related quality of life and associated factors in Chinese patients undergoing haemodialysis. Health Qual Life Outcomes. 2015 Jul 29;13:108.

6. Wang R, Tang C, Chen X, Zhu C, Feng W, Li P, Lu C. Poor sleep and reduced quality of life were associated with symptom distress in patients receiving maintenance hemodialysis. Health Qual Life Outcomes. 2016 Sep 8;14(1):125.

7. Yang YJ, Yuan HH, Xue GF. Current status and influencing factors of health related quality life inyoung patients with maintenance hemodialysis. Chinese Nursing Research, 2020,34(11):2016-2019.

8. Deng FY. Construction and application of "Internet+" transitional nursing intervention program for hemodialysis patients based on the opportunity theory.MNS. Thesis. University of South China,2019.

9. Liang JL.Current status of research on quality of life assessment and influencing factors of maintenance hemodialysis patients.Internal Medicine,2013,8(02):191-193.

10. Ai B, Wang S, Xing DE. Study on the relationship between the subjective health perception and survival time of the elderly--Based on the 9-year tracking data of the urban elderly in Shenyang.Chinese Journal of Health 
Statistics,2015,32(05):875-877 + 880 .

11. Park JI, Park TW, Yang JC, Chung SK. Factors associated with depression among elderly Koreans: the role of chronic illness, subjective health status, and cognitive impairment. Psychogeriatrics. 2016 Jan;16(1):62-9.

12. Power, M. Development of a common instrument for quality of life. In: Nosikov A, Gudex C, editors. EUROHIS: Developing common instruments for health surveys. Amsterdam: los Press; 2003. p. 145-59.

13. Yang F, Wong $\mathrm{C} \mathrm{K} \mathrm{H}$, Lou N, et al. Mapping the kidney disease quality of life 36-item short form survey(KDQOL36) to the EQ-5D-3L and the EQ-5D-5L in patients undergoing dialysis. Eur J Health Econ,2019,20(8):11951206.

14. Tao X, Chow SK, Wong FK. Determining the validity and reliability of the Chinese version of the Kidney Disease Quality of Life Questionnaire (KDQOL-36 ${ }^{\mathrm{TM}}$ ). BMC Nephrol. 2014 Jul 11;15:115.

15. Gutiérrez-Peredo GB, Martins MTS, da Silva FA, Lopes MB, Lopes GB, Lopes AA. Functional dependence and the mental dimension of quality of life in Hemodialysis patients: the PROHEMO study. Health Qual Life Outcomes. 2020 Jul 17;18(1):234.

16. Wang GW, Liu SY, Zhou J, Luo LM. A cross-sectional study on the survival status of single-center maintenance hemodialysis patients.Shanghai Medical Journal,2020,43(12):740-744.

17. He MX, Yang YJ, Yu SB. Correlation analysis of quality of life and health literacy in middle-aged and elderly patients undergoing maintenance hemodialysis.Chinese Nursing Research,2020,34(21):3886-3889.

18. Yuan H, Zhang Y, Xue G, Yang Y, Yu S, Fu P. Exploring psychosocial factors associated with frailty incidence among patients undergoing maintenance hemodialysis. J Clin Nurs. 2020 May;29(9-10):1695-1703.

19. Yang Z, Huang YL, Lu HZ, Zou BL, Wang H, Chen GW, Wu YP. Study on influencing factors of dietary status of patients with maintenance hemodialysis and correlation with quality of life.Chinese Nursing Research,2021,35(14):2560-2564.

20. Liu MJ, Cui WP, Miao LN, Zhao J, Zhou WH. Advances in the Researches of Somnipathy in Patients Underwent Hemodialysis.Chinese General Practice,2020,23(29):3712-3718 + 3728.

21. He YH, Jiang YF. Symptom experiences among maintenance hemodialysis patients: a cross-sectional study.Chinese Journal of Nursing,2016,51(3):299-303.

22. Evangelidis N, Tong A, Manns B, et al. Developing a set of core outcomes for trials in hemodialysis:an international delphi survey. Am J Kidney Dis,2017,70(4):464-475.

23. Bossola M, Tazza L. Postdialysis Fatigue: A Frequent and Debilitating Symptom. Semin Dial. 2016 May;29(3):222-7.

24. Yao Q, Tan ZJ. Clinical effect of hemodialysis combined with hemoperfusion in the treatment of uremic pruritus.Contemporary Medicine,2018,24(27):151-153.

25. Makkar V, Kumar M, Mahajan R, Khaira NS. Comparison of Outcomes and Quality of Life between Hemodialysis and Peritoneal Dialysis Patients in Indian ESRD Population. J Clin Diagn Res. 2015 Mar;9(3):0C28-31.

26. Bai YH, Yang M, Liao YJ, Jiang HY, Feng ZJ. Mental status and quality of life of maintenance hemodialysis patients and their influencing factors.Chinese Journal of Gerontology,2018,38(03):728-730.

27. Chen YH, Ma WG. Investigation on the quality of life of patients with end-stage renal disease on maintenance hemodialysis.International Journal of Nursing,2015,34(17):2321-2324.

28. Du CY, Wu SS, Lin XH, Shang YB, Teng S, Liu HX. Research progress on the level and influencing factors of quality of life in patients with maintenance hemodialysis.Journal of Nursing Administration,2016,16(07):485487.

Page $16 / 19$ 
29. Erickson KF, Zhao B, Ho V, Winkelmayer WC. Employment among Patients Starting Dialysis in the United States. Clin J Am Soc Nephrol. 2018 Feb 7;13(2):265-273.

30. HUANG B, LAI B, XU L, et al. Low employment and low willingness of being reemployed in Chinese working-age maintained hemodialysis patients. Renal Failure,2017,39(1):607-612.

31. Li X, Sun XJ. Self-perceived burden and related factors in patients with maintenance hemodialysis. Chinese Mental Health Journal,2016,30(04):258-263.

32. Ribeiro MCCB, Vogt BP, Vannini FCD, Caramori JCT. Role of parathyroid hormone in anorexia on maintenance hemodialysis patients. Clin Nutr ESPEN. 2019 Dec;34:137-141.

33. Li JH, Huang LZ, Ren XH, Zhao J, Tang MM, Ren L. Correlation between symptom burden and sleep quality, quality of life in Maintenance Hemodialysis patients.Chinese Nursing Management,2016,16(01):25-29.

\section{Figures}

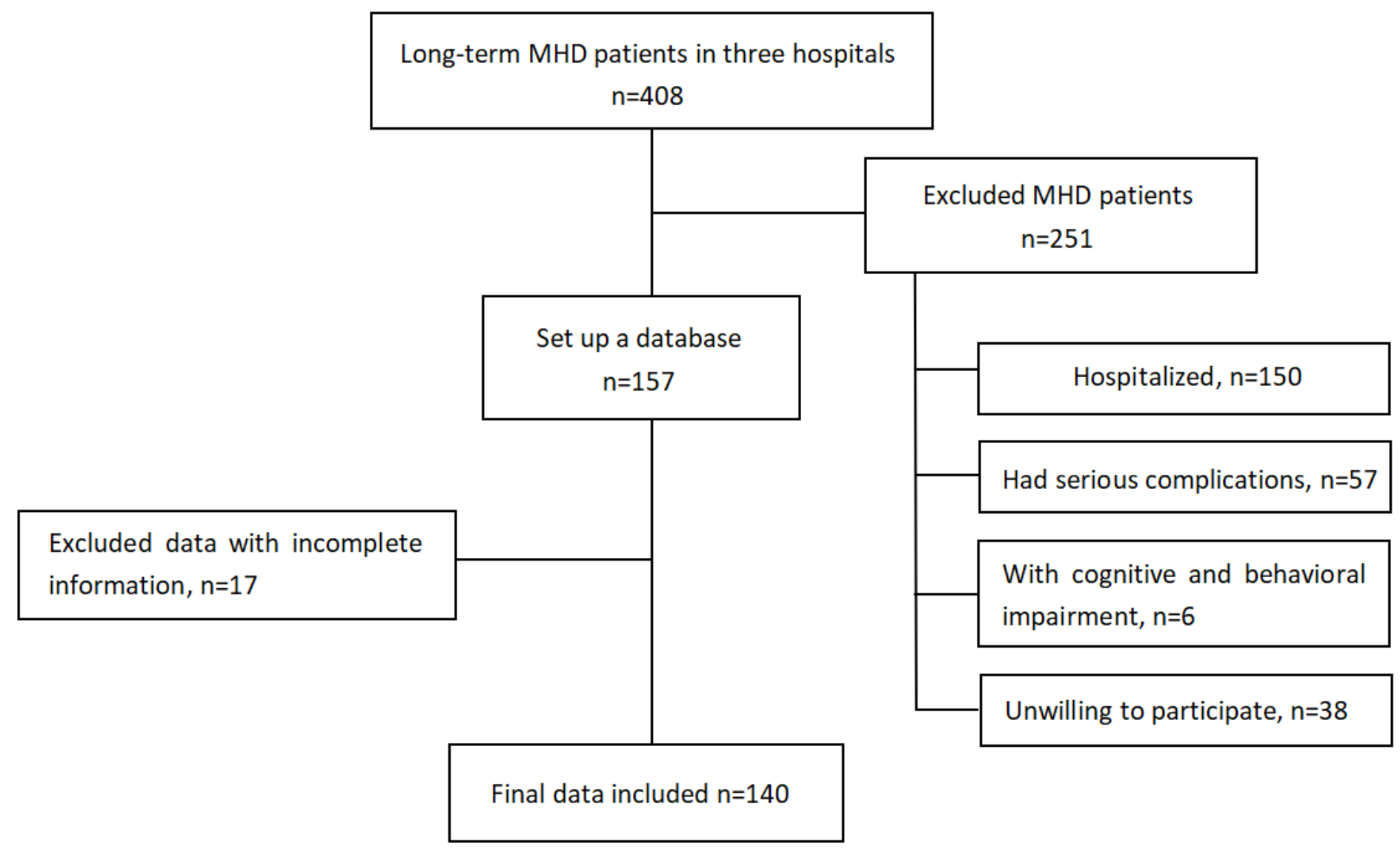

Figure 1

Flowchart of study participants 


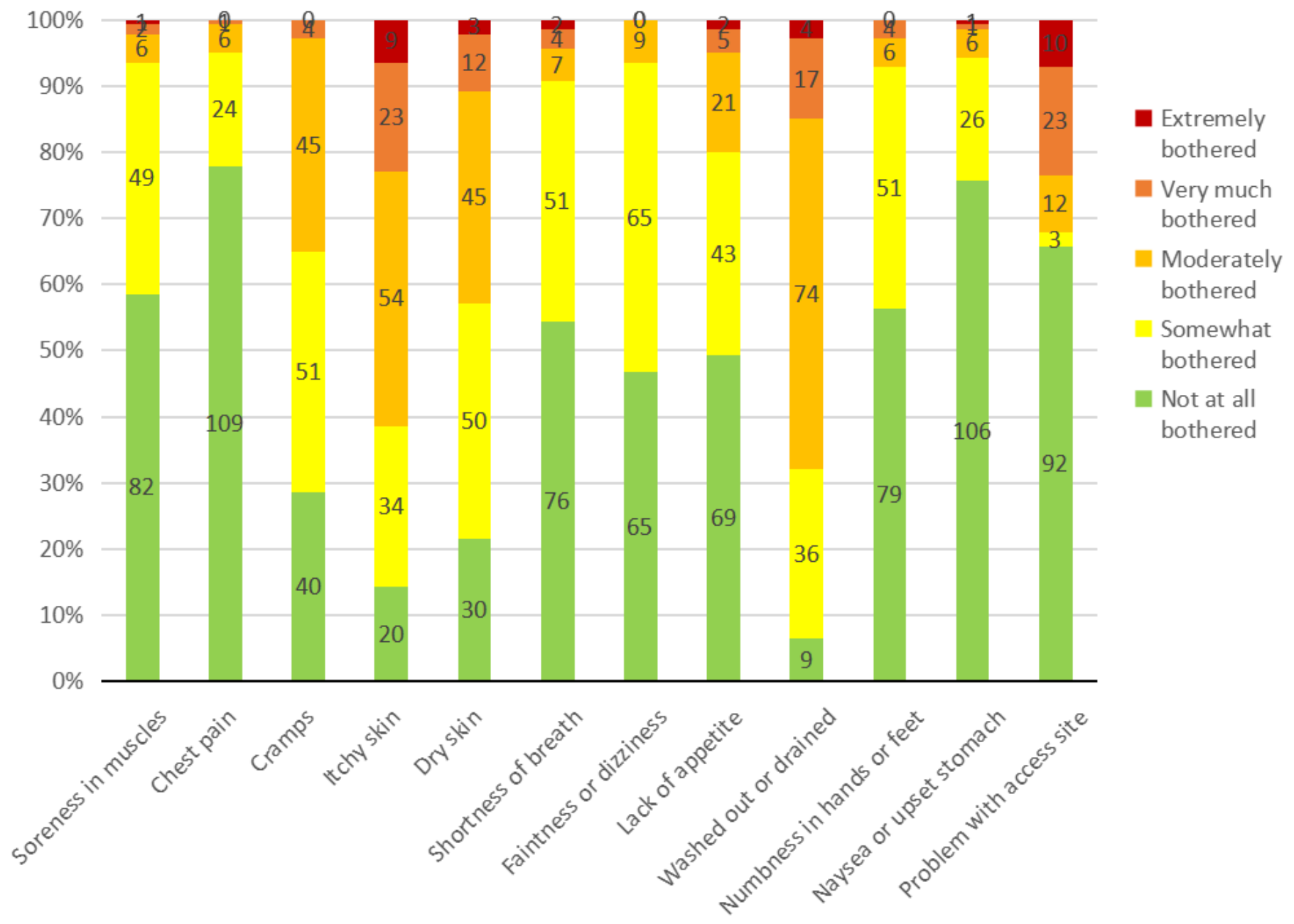

Figure 2

Kidney disease-related symptoms 


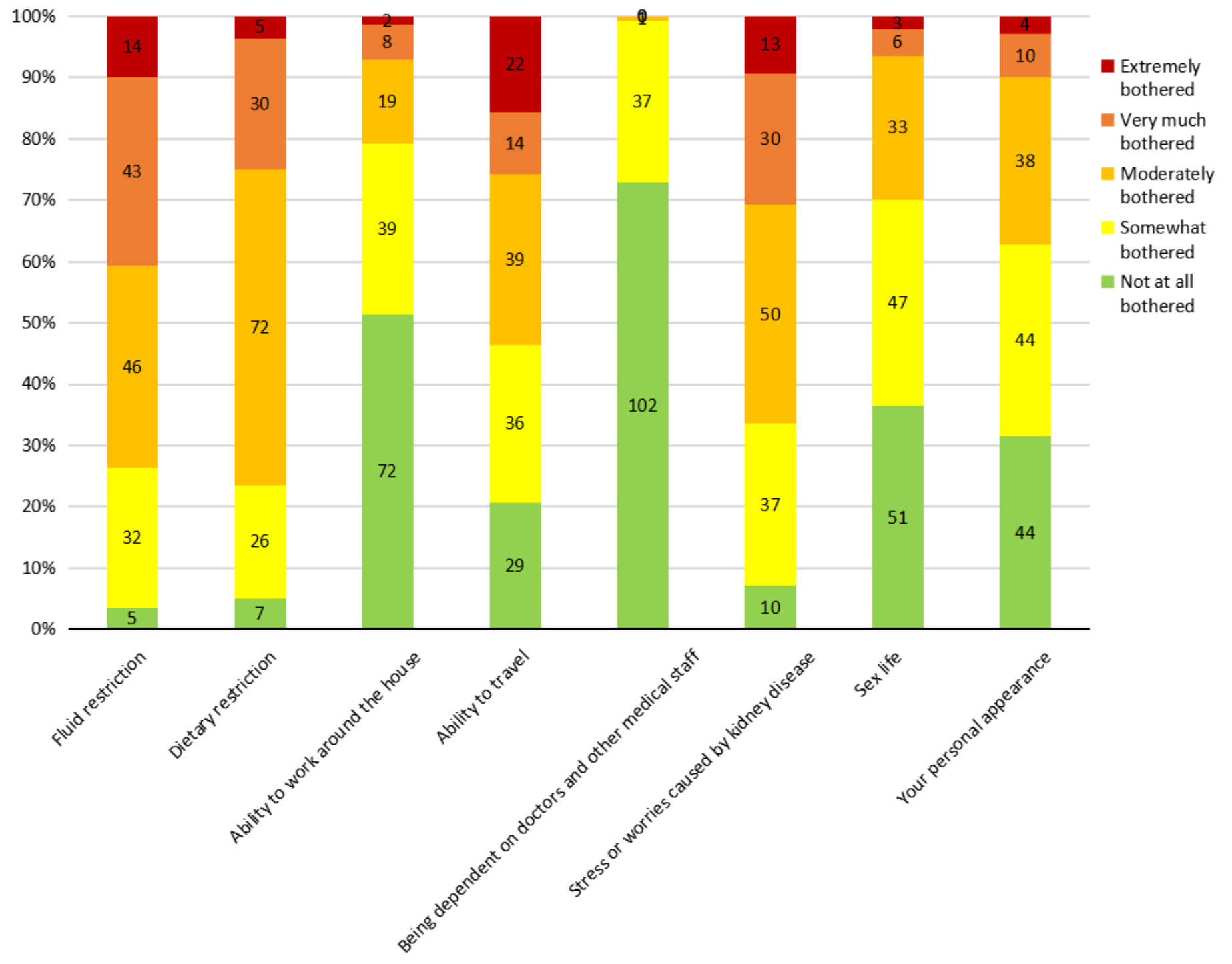

Figure 3

Kidney disease impact 